\title{
A NEW METHOD FOR MEASURING EXTRAGALACTIC DISTANCES
}

\author{
Yuzuru Yoshit ${ }^{1,5}$, Yukiyasu Kobayashi ${ }^{2}$, Takeo Minezaki ${ }^{1}$, Shintaro Koshida ${ }^{3}$, and Bruce A. Peterson ${ }^{4}$ \\ ${ }^{1}$ Institute of Astronomy, School of Science, University of Tokyo, 2-21-1 Osawa, Mitaka, Tokyo 181-0015, Japan; yoshii@ ioa.s.u-tokyo.ac.jp \\ ${ }^{2}$ National Astronomical Observatory, 2-21-1 Osawa, Mitaka, Tokyo 181-8588, Japan \\ ${ }^{3}$ Center of Astro Engineering and Department of Electrical Engineering, Pontificia Univercsidad Catolica de Chile, Av. Vicuna Mackenna 4868, Chile \\ ${ }^{4}$ Mount Stromlo Observatory, Research School of Astronomy and Astrophysics, Australian National University, Weston Creek P.O., ACT 2611, Australia \\ Received 2013 October 15; accepted 2014 February 23; published 2014 March 7
}

\begin{abstract}
We have pioneered a new method for the measurement of extragalactic distances. This method uses the time lag between variations in the short wavelength and long wavelength light from an active galactic nucleus (AGN), based on a quantitative physical model of dust reverberation that relates the time lag to the absolute luminosity of the AGN. We use the large homogeneous data set from intensive monitoring observations in optical and near-infrared wavelength bands with the dedicated $2 \mathrm{~m}$ MAGNUM telescope to obtain the distances to 17 AGNs in the redshift range $z=0.0024$ to $z=0.0353$. These distance measurements are compared with distances measured using Cepheid variable stars, and are used to infer that $H_{0}=73 \pm 3$ (random) $\mathrm{km} \mathrm{s}^{-1} \mathrm{Mpc}^{-1}$. The systematic error in $H_{0}$ is examined, and the uncertainty in the size distribution of dust grains is the largest source of the systematic error, which is much reduced for a sample of AGNs for which their parameter values in the model of dust reverberation are individually measured. This AGN time lag method can be used beyond $30 \mathrm{Mpc}$, the farthest distance reached by extragalactic Cepheids, and can be extended to high-redshift quasi-stellar objects.
\end{abstract}

Key words: cosmological parameters - dust, extinction - galaxies: active - galaxies: Seyfert

Online-only material: color figures

\section{INTRODUCTION}

Hubble (1929) discovered that the universe is expanding by finding a correlation between a galaxy's recession velocity and its distance. Since then, a reliable estimate of the expansion rate of the universe at the current epoch has been a central subject in observational cosmology. This expansion rate is denoted by the Hubble constant, $H_{0}$, and it characterizes the nature of the universe, such as the age of the universe, $t_{0}=1 / H_{0}$, the observable size of the universe, $R_{0}=c / H_{0}$, and the critical mass density of the universe, $\rho_{\text {crit }, 0}=3 H_{0}^{2} /(8 \pi G)$, where $c$ is the speed of light, and $G$ is the gravitational constant.

A variety of empirical distance-ladder methods have been proposed, which determine the distance to a galaxy through a series of steps, with each step calibrating the next more distant step, such as those with final steps based upon the period-luminosity relation for Cepheid variable stars, the maximum luminosity of type Ia supernovae, etc. The results from these empirical methods have almost converged to a value of $H_{0}$ at around $73 \mathrm{~km} \mathrm{~s}^{-1} \mathrm{Mpc}^{-1}$ (Freedman et al. 2001; Freedman \& Madore 2010).

On the other hand, a physical method has the advantage over the empirical methods in that it could, in principle, determine $H_{0}$ through a reasonable model parameterization without resorting to an empirical distance ladder. The Sunyaev-Zel'dovich effect (Birkinshaw 1999) and the use of gravitational lensing (Blandford \& Narayan 1986) have been proposed. However, their results for $H_{0}$ have not converged to agree with those from the empirical methods, because uncertainties associated with parameters in these particular methods do not allow an accuracy comparable to the empirical methods (Freedman \& Madore 2010). Consequently, other physical methods that enable the measurement of extragalactic distances with higher accuracy are eagerly sought.

\footnotetext{
5 PI of the MAGNUM project.
}

In this Letter, we propose a new physical method, using a model with simple physics, for extragalactic distance determination. Our model is based on the dust reverberation in active galactic nuclei (AGNs). We demonstrate the effectiveness of this method by determining $H_{0}$ with a value and accuracy comparable to that obtained by the Hubble Key Project using Cepheid variable stars.

\section{METHOD AND OBSERVATIONAL DATA}

An AGN consists of a hot central engine surrounded by a cooler dust torus (e.g., Antonucci 1993; Urry \& Padovani 1995). Dust near the central engine is sublimated, creating a gap between the central engine and the inner radius of the torus. Dust grains beyond the sublimation radius, $r_{\mathrm{d}}$, absorb short wavelength light produced by the central engine, and re-emit the absorbed energy at long wavelengths. Variations in the flux emitted by the central engine are mimicked at long wavelengths by the dust torus after a time delay, $\Delta t=r_{\mathrm{d}} / c$, corresponding to the light travel time between the central engine and the inner radius of the dust torus. Apparently, $r_{\mathrm{d}}$ is determined by the absolute luminosity, $L$, of the central engine according to $r_{\mathrm{d}} \propto L^{1 / 2}$ (Barvainis 1987; Oknyanskij \& Horne 2001; Minezaki et al. 2004; Suganuma et al. 2006). Thus, by measuring the time delay, $\Delta t$, we can obtain the absolute luminosity of the AGN, and its distance.

We assume local energy balance between the absorbed short wavelength ultraviolet (UV) radiation and the re-emitted long wavelength near-infrared (NIR) radiation at the inner radius of the dust torus:

$$
\pi a^{2} \int_{\mathrm{UV}} Q_{\nu}(a) \frac{L_{v}}{4 \pi r_{\mathrm{d}}^{2}} d v=4 \pi^{2} a^{2} \int_{\mathrm{NIR}} Q_{\nu}(a) B_{\nu}\left(T_{\mathrm{d}}\right) d v,
$$

where $L_{v}=\left(v / v_{V}\right)^{\alpha_{\mathrm{UV}}} L_{V}$ in the UV to optical region with the power-law index $\alpha_{\mathrm{UV}}, L_{V}$ is the absolute luminosity in the $V$-band, $B_{v}\left(T_{\mathrm{d}}\right)$ is the Plank function for the characteristic 


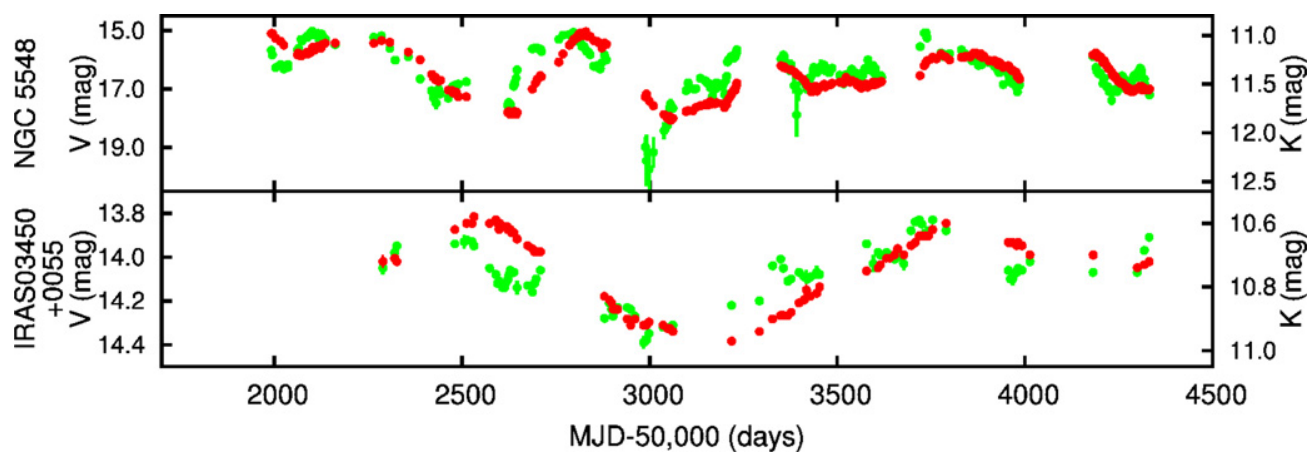

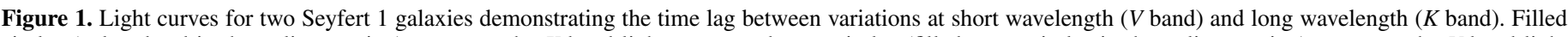

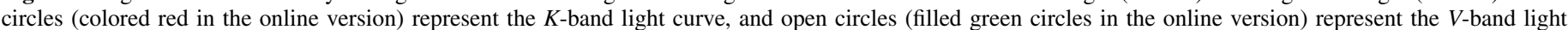

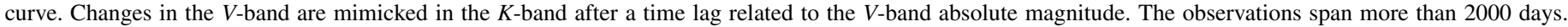
(a) Observations of NGC 5548 in the $V$ and $K$ bands. (b) Observations of IRAS $03450+0055$ in the $V$ and $K$ bands.

(A color version of this figure is available in the online journal.)

dust sublimation temperature $T_{\mathrm{d}}$, and $Q_{v}(a)$ is the absorption coefficient per dust grain of mean radius $a$ as a function of $v$ taken from the literature (Draine \& Lee 1984; Laor \& Draine 1993). Values of the three parameters, $\alpha_{\mathrm{UV}}, T_{\mathrm{d}}$, and $a$, are set using observations of AGN. We have $\alpha_{\mathrm{UV}}=-0.5 \pm 0.2$ taken from a composite QSO spectrum (Vanden Berk et al. 2001; Davis et al. 2007). We adopt a rather large uncertainty of \pm 0.2 which well covers the observed range of QSO to QSO spectral index variation. With this $\alpha_{\mathrm{UV}}$, we truncate the UV spectrum at $0.03 \mu \mathrm{m}$, considering the UV turnover of QSO spectrum (e.g., Telfer et al. 2002; Krawczyk et al. 2013) and the decrease of dust absorption efficiency at shorter wavelength below this truncation (Draine \& Lee 1984; Laor \& Draine 1993). We have $T_{\mathrm{d}}=1700 \pm 50 \mathrm{~K}$ evaluated from the $H-K$, $J-K$, and $J-H$ color temperatures of the variable NIR component for a sample of Seyfert 1 galaxies observed with the MAGNUM telescope (Tomita 2005; Tomita et al. 2006). ${ }^{6}$ The value for $T_{\mathrm{d}}$ is consistent with the condensation temperature for solid carbon (Huffman 1997; Salpeter 1977). Since dust grains other than graphite grains sublimate below this temperature, the absorption coefficient of graphite grains is adopted for $Q_{v}(a)$. The distribution of $a$ is assumed to have a powerlaw form of $f(a)=K a^{-p}$ with parameters of $p=2.75$, $a_{\min }=0.005 \mu \mathrm{m}$, and $a_{\max }=0.20 \mu \mathrm{m}$ (cf. Section 3). The mean grain size for this distribution is larger than that in the interstellar medium, based on the analysis of UV extinction curves of radio-quiet AGNs (Gaskell et al. 2004).

In order to obtain $\Delta t_{\mathrm{AGN}}$ in the rest frame of the AGN, two cosmological effects must be considered. The observed $\Delta t$ must be corrected for time dilation, a factor of $(1+z)^{-1}$, where $z$ is the redshift. We also consider an effect that arises from the temperature gradient in the dust torus and the shift of the $K$-band, where our observations are made, to a different wavelength in the reference frame of the AGN according to $\lambda_{\mathrm{AGN}}=\lambda_{K} /(1+z)$. At higher redshifts, we observe shorter

\footnotetext{
6 Since the time delay is measured using the NIR to optical flux variations, the dust temperature near $r_{\mathrm{d}}$ is evaluated from the NIR color temperatures of the variable component rather than the average or single-epoch NIR spectral energy distributions of the whole component which contains the contribution from the dust of lower temperatures at larger radii beyond $r_{\mathrm{d}}$ (e.g., Kishimoto et al. 2007; Landt et al. 2011). AGNs in our sample show no burst-like flux variation but repeat the brighter and fainter states in turn (Figure 1), keeping their NIR colors almost constant (Tomita 2005; Suganuma et al. 2006). Therefore, the dust temperature of the variable component is regarded as staying almost constant during the flux variation. Note that the color temperatures can be determined without subtracting the host-galaxy flux, which would often be an origin of large uncertainty.
}

wavelengths emitted by the AGN from a hotter part of the dust torus that is closer to the central engine. This effect makes the observed $\Delta t$ shorter than would be the case if the same AGN was observed at a smaller redshift. We formulate the correction to our $K$-band observations empirically, by using the mean delay of $H$-band to $K$-band, which is about 0.3 times the delay of $V$-band to $K$-band, as obtained from MAGNUM telescope observations of nearby Seyfert 1 galaxies (Tomita et al. 2006). Interpolating this band-dependent delay linearly with redshift, and correcting for the time dilation, we obtain the correction formula $\Delta t_{\mathrm{AGN}}=\Delta t(1+1.24 z) /(1+z)$. For the redshift range in this Letter, $z \leqslant 0.035$, the combined corrections are less than $1 \%$ and are unimportant.

The MAGNUM project was initiated in order to measure $\Delta t$ for AGNs over a range of redshifts (Yoshii 2002; Yoshii et al. 2003). The observations were made with a multicolor imaging photometer (Kobayashi et al. 1998) mounted on a dedicated $2 \mathrm{~m}$ telescope located at the summit of Haleakala on the Hawaiian Island of Maui. Monitoring observations from 2001 through 2007 were made simultaneously in two wavelength bands, the optical $V(0.55 \mu \mathrm{m})$ band and the NIR $K(2.2 \mu \mathrm{m})$ band, to determine $\Delta t$. Variations in the short wavelength flux which heats the dust grains is synchronous with variations in the $V$-band (e.g., Winkler 1997; Sakata et al. 2010) and dust near the sublimation temperature emits the most energy in the $K$-band (e.g., Barvainis 1990; Kobayashi et al. 1993). In Figure 1, we present light curves in the $V$-band and $K$-band for NGC 5548 and IRAS 03450+0055, as examples. The flux variations in the $V$-band and $K$-band resemble each other in shape, with the $K$-band flux variations delayed with respect to those in the $V$-band. In this Letter, we use the time-delay data for 17 Seyfert 1 galaxies obtained by the MAGNUM project. We only briefly describe the procedures of our data reduction and analysis here, because the details will be presented elsewhere (S. Koshida et al., in preparation).

The time delay, $\Delta t$, was obtained by cross-correlating the $V$-band and $K$-band light curves using a bi-directional interpolation scheme which weights the observed flux data more than a simulated interpolation scheme with respect to time (Suganuma et al. 2006).

Before taking the cross correlation between the $V$-band and $K$-band light curves, we subtracted the accretion disk component from the observed $K$-band flux to derive the dust torus component in the $K$ band (Koshida et al. 2009). Emission from the central accretion disk extends into the NIR wavelengths (Minezaki et al. 2006; Tomita et al. 2006; Kishimoto et al. 2008). 
Table 1

List of Target Active Galactic Nuclei

\begin{tabular}{|c|c|c|c|c|c|c|c|c|}
\hline Object & R.A. & Decl. & $z^{\mathrm{a}}$ & $\begin{array}{c}v^{\mathrm{b}} \\
\left(\mathrm{km} \mathrm{s}^{-1}\right)\end{array}$ & $\begin{array}{l}m_{V}^{\mathrm{c}} \\
(\mathrm{mag})\end{array}$ & $\begin{array}{c}A_{V}{ }^{\mathrm{d}} \\
(\mathrm{mag})\end{array}$ & $\begin{array}{c}\Delta t^{\mathrm{e}} \\
\text { (days) }\end{array}$ & $\begin{array}{c}d^{\mathrm{f}} \\
(\mathrm{Mpc})\end{array}$ \\
\hline Mrk 335 & 000619.5 & +201210.5 & 0.0258 & $8996.07 \pm 1383.98$ & $14.59 \pm 0.02$ & 0.118 & $139.2 \pm 16.4$ & $145.6 \pm 17.3$ \\
\hline Mrk 590 & 021433.6 & -004600.1 & 0.0264 & $7176.93 \pm 551.52$ & $16.56 \pm 0.08$ & 0.124 & $36.8 \pm 2.7$ & $95.2 \pm 7.0$ \\
\hline IRAS $03450+0055$ & 034740.2 & +010514.0 & 0.0310 & $7787.03 \pm 1347.99$ & $14.74 \pm 0.02$ & 0.660 & $103.3 \pm 4.7$ & $90.4 \pm 4.1$ \\
\hline Akn 120 & 051611.4 & -000859.4 & 0.0327 & $8041.23 \pm 1695.01$ & $13.82 \pm 0.02$ & 0.426 & $135.4 \pm 16.8$ & $86.5 \pm 10.8$ \\
\hline MCG -08-11-011 & 055453.5 & +462622.0 & 0.0205 & $6541.04 \pm 331.25$ & $15.11 \pm 0.06$ & 0.720 & $92.6 \pm 13.7$ & $93.1 \pm 13.9$ \\
\hline Mrk 79 & 074232.8 & +494834.8 & 0.0222 & $6753.90 \pm 75.14$ & $15.11 \pm 0.04$ & 0.235 & $71.9 \pm 3.3$ & $90.5 \pm 4.2$ \\
\hline Mrk 110 & 092512.9 & +521710.5 & 0.0353 & $10240.19 \pm 587.93$ & $15.28 \pm 0$ & 0.043 & 88.5 & $132.5 \pm 9.1$ \\
\hline NGC 3 & 102330.6 & +195154.0 & 0.0039 & $1214.99 \pm 35.56$ & $14.56 \pm$ & 0.075 & 13.9 & $14.5 \pm 0.6$ \\
\hline NGC & 11064 & +72340 & 0.0 & $3836.38 \pm$ & 15.09 & 40 & 51.2 & $66.1=$ \\
\hline Mrk 744 & 1139 & +31543 & 0.0 & $4171.02 \pm$ & $17.23=$ & 0.079 & 20.9 & $74.3 \pm 7.8$ \\
\hline NGC 4051 & 1203 & +443152.8 & 0.0023 & $901.24 \pm$ & $14.85 \pm$ & 0.043 & $14.6=$ & $17.6 \pm 0.6$ \\
\hline NGC 4151 & 121032.6 & +392420.6 & 0.0033 & $1409.56 \pm$ & $13.45 \pm 0.09$ & 0.092 & $47.2 \pm 0.7$ & $29.2 \pm 0.4$ \\
\hline NGC 4593 & 123939.4 & -052039.4 & 0.0090 & $3540.76 \pm 545.81$ & $15.23 \pm 0.04$ & 0.082 & $43.6 \pm 1.9$ & $61.9 \pm 2.7$ \\
\hline NGC 5548 & 141759.5 & +2508 12.4 & 0.0172 & $6115.16 \pm 486.34$ & $15.81 \pm 0.03$ & 0.068 & $49.5 \pm 0.8$ & $92.5 \pm 1.5$ \\
\hline Mrk 817 & 143622.1 & +584739.4 & 0.0315 & $8779.59 \pm 1023.15$ & $14.83 \pm 0.02$ & 0.022 & $87.9 \pm 8.1$ & $107.6 \pm 10.0$ \\
\hline Mrk 509 & 204409.7 & -104324.5 & 0.0344 & $9026.24 \pm 1419.98$ & $13.87 \pm 0.03$ & 0.190 & $144.5 \pm 9.1$ & $105.4 \pm 6.7$ \\
\hline NGC 7469 & 230315.6 & +085226.4 & 0.0163 & $3967.24 \pm 906.33$ & $14.58 \pm 0.06$ & 0.228 & $48.4 \pm 1.5$ & $47.7 \pm 1.5$ \\
\hline
\end{tabular}

Notes.

${ }^{a}$ The heliocentric redshift from the NASA/IPAC Extragalactic Database (NED).

${ }^{\mathrm{b}}$ The the cosmic recession velocity: $c z$ corrected for galactic rotation and the local velocity flow. The \pm figures represent the uncertainty in the models of the local velocity flow.

${ }^{\mathrm{c}}$ The mean of the $V$-band fluxes generated at equal intervals of time, after excluding the data which statistically show no flux variation in the time bins, with the $1 \sigma$ error.

d The galactic extinction correction for $m_{V}$.

${ }^{\mathrm{e}}$ The time lag with $1 \sigma$ errors as described in the text.

${ }^{\mathrm{f}}$ The distance, $d$, as determined from Equation (2).

Variations of this component appear in the $K$ band, and make a measured $\Delta t$ shorter than the light travel time. We correct by estimating the contaminating flux as $f_{K}=\left(v_{V} / \nu_{K}\right)^{\alpha_{V K}} f_{V}$ from the simultaneous $V$-band measurement, and subtracting it from the $K$-band measurement. We use $\alpha_{V K}=0.1 \pm 0.11$ obtained from a cross multiple regression analysis of our sample of nearby Seyfert 1 galaxies (Tomita 2005; Tomita et al. 2006).

In the light curves of 17 Seyfert 1 galaxies, we found 49 single incidents of a flux maximum or minimum which could be used to measure $\Delta t$. We give the mean value of $\Delta t$ for those galaxies which underwent several incidents. In Table 1 we give, for each of the 17 Seyfert 1 galaxies that we monitored, our mean observed apparent magnitude, $m_{V}$, the time delay, $\Delta t$ in days, and the luminosity distance, $d$ in Mpc, from Equation (2)

$$
d=\Delta t_{\mathrm{AGN}} \times 10^{0.2\left(m_{V}-A_{V}-k_{V}-25+g\right)},
$$

where $A_{V}$ is Galactic extinction (Schlegel et al. 1998), $k_{V}$ is the $K$-correction, and $g=10.60$. The calibration factor, $g$, was calculated from Equation (1) using the physical model with the parameters described above. The $K$-correction was calculated assuming a power-law spectrum in the UV to optical range, $f_{v} \propto v^{\alpha_{\mathrm{UV}}}$, to obtain $k_{V}=-2.5\left(1+\alpha_{\mathrm{UV}}\right) \log (1+z)$, where $\alpha_{\mathrm{UV}}=-0.5$.

The heliocentric redshift, $z$, is taken from published H I measurements, the cosmic recession velocity, $v$, is the heliocentric velocity, $c z$, corrected for galactic rotation and the local velocity flow using the average of two different models of the local velocity flow (Mould et al. 2000; Tonry et al. 2000), as was done for the Cepheid distance determinations (Freedman et al. 2001). The \pm figures given for $v$ in Table 1 represent the uncertainty in the flow models and are half the difference between the correction to $v$ obtained from the two models.

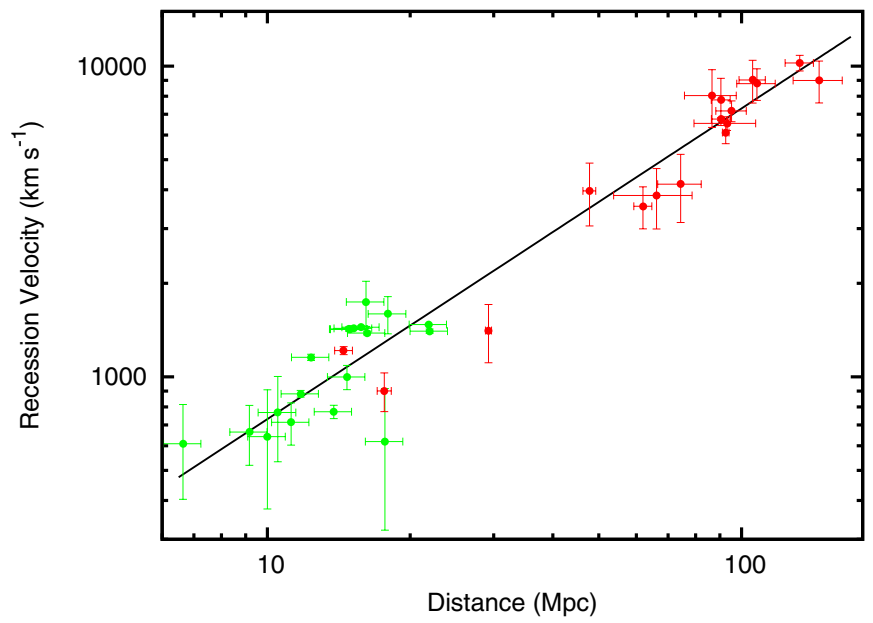

Figure 2. Velocity vs. distance for galaxies with distances measured using Cepheid variable stars and for galaxies with distances measured using the AGN time lag method in this Letter. Open circles (filled green circles in the online version) represent the data based on Cepheid variable stars as part of the Hubble Space Telescope Key Project (Freedman et al. 2001), and filled circles (colored red in the online version) represent our results. All recession velocities have been derived from redshift measurements that have been corrected for the local velocity field using the same procedures. The inclined line represents the relation $v=H_{0} d$ where $H_{0}=73 \mathrm{~km} \mathrm{~s}^{-1} \mathrm{Mpc}^{-1}$. The horizontal error bars represent $1 \sigma$ errors in the distance. The vertical error bars represent the uncertainty in the local velocity field correction.

(A color version of this figure is available in the online journal.)

\section{RESULTS AND DISCUSSION}

In Figure 2, we present the velocity-distance diagram to compare the extragalactic distances measured using our AGN time lag method with distances obtained from Cepheid variable 
stars. Our AGN time lag method determines the distance, $d$, through a reasonable physical parameterization, and directly provides the first step in the distance ladder. We obtain the Hubble constant, $H_{0}=73 \pm 3 \mathrm{~km} \mathrm{~s}^{-1} \mathrm{Mpc}^{-1}$, from a least squares fit to $v=H_{0} d$ using our AGN time lag distances. The Hubble constant found from empirically calibrated Cepheid distances is $H_{0}=75 \pm 10$ (Freedman et al. 2001). Our AGN time lag method, which is based upon a physical model with no empirical calibration, agrees well with the Cepheid distances and other empirical distance ladders (Freedman et al. 2001; Freedman \& Madore 2010), but extends to galaxies 10 times more distant than where Cepheid distances can be measured, to where the cosmic recession velocity is not so badly afflicted by the local velocity flow.

The systematic error in $H_{0}$ is estimated by changing the parameter values of $\alpha_{\mathrm{UV}}$ and $T_{\mathrm{d}}$ in their respective ranges of uncertainty, and by changing the distribution of $a$, assumed to have a power-law form of $f(a)=K a^{-p} .{ }^{7}$ Our calculation gives $\Delta H_{0, \alpha}= \pm 5 \mathrm{~km} \mathrm{~s}^{-1} \mathrm{Mpc}^{-1}$ and $\Delta H_{0, T_{\mathrm{d}}}= \pm 3 \mathrm{~km} \mathrm{~s}^{-1} \mathrm{Mpc}^{-1}$ for the uncertainty in $\alpha_{\mathrm{UV}}$ and $T_{\mathrm{d}}$, respectively. The systematic error in $H_{0}$ from the uncertainty in $f(a)$ is estimated using two extreme size distributions, such as the standard MRN distribution in the local interstellar medium for our Galaxy $\left(p=3.5, a_{\min }=0.005 \mu \mathrm{m}, a_{\max }=0.25 \mu \mathrm{m}\right.$; Mathis et al. 1997), and the larger grain enhanced distribution for AGNs $\left(p=2.05, a_{\min }=0.005 \mu \mathrm{m}, a_{\max }=0.20 \mu \mathrm{m}\right)$, because smaller dust grains are more efficiently sublimated by UV radiation from the central engine (Gaskell et al. 2004). We adopt an intermediate value of $p=2.75$ with $a_{\min }=0.005 \mu \mathrm{m}$ and $a_{\max }=0.20 \mu \mathrm{m}$ as our standard grain size distribution. The NIR flux-weighted averaging scheme over the full range of $a$ is then applied to Equation (1), and the calibration factor $g$ is calculated for the two extreme distributions as well as the intermediate one. The uncertainty in $g$ is within the limits of $\Delta g= \pm 0.5$, so that $\Delta H_{0, a}$ is at most \pm 0.1 dex from Equation (2), which is currently the largest source of the systematic error in $H_{0}$. This error in $H_{0}$ is much reduced for a sample of AGNs for which their $\alpha_{\mathrm{UV}}, T_{\mathrm{d}}$, and $f(a)$ are individually measured, because the target to target variation of these parameters also contributes to the random error in the $H_{0}$ fitting. In particular, if monitoring observations of high-redshift AGNs were obtained, their UV-optical spectrum could be determined directly from ground based spectroscopic observations, and the accuracy of the $H_{0}$ determination would be significantly improved. Alternatively, if many of target AGNs were calibrated by other reliable distance indicators such as Cepheids and type Ia supernovae, their calibrated distances would not only provide a cross check on our method of distance determination, but also independently constrain the parameter values in a physical model of dust reverberation.

Two other reverberation methods of distance determination of AGN have been proposed. One is a method based on the reverberation mapping of the broad-line emitting region (BLR) in AGN (Watson et al. 2011; Czerny et al. 2013), using an empirical relation between the radial distance of BLR from the AGN center and the AGN luminosity. Although the radiusluminosity relation of BLR is well established now (Bentz et al. 2013), the exact size of BLR cannot be predicted theoretically. Therefore, this method remains as an empirical method in the

\footnotetext{
7 The observer's viewing angle has also been considered as a possible source of the systematic error (e.g., Kawaguchi \& Mori 2011). However, we do not explicitly consider this possibility here, because we have not found any systematic difference in the time lag measurements for our target AGNs of different Seyfert subclasses (S. Koshida et al., in preparation).
}

sense that it must use empirical distance-ladder methods for calibration.

Another is a method based on the wavelength-dependent time delay of UV-optical continuum emission from the central accretion disk in AGNs (Collier et al. 1999; Cackett et al. 2007). This method is a physical one, like our method, which could, in principle, determine the distance without any distance calibration. However, this method gives $H_{0}=42-44 \mathrm{~km} \mathrm{~s}^{-1} \mathrm{Mpc}^{-1}$, which is about a factor of 1.7 smaller than current standard estimates, and even below the recent lower estimate of $67 \mathrm{~km} \mathrm{~s}^{-1} \mathrm{Mpc}^{-1}$ based on Planck measurements of the cosmic microwave background temperature (Planck Collaboration et al. 2014). Thus, this method seems to have some difficulties to be resolved, such as the adequacy of modeling the X-ray reprocessing for the flux variation of UV-optical continuum emission. In fact, while some AGNs show a delay of the optical flux variation behind that of X-ray, which is consistent with the X-ray reprocessing, some others show a delay of the X-ray variation behind that of optical, or they show a poor correlation between the X-ray and optical variations (e.g., Uttley 2006).

In summary, we have demonstrated that our AGN time lag method can be used to measure extragalactic distances beyond what is possible with Cepheids, and we have obtained a value of the Hubble constant with our AGN time lag method of $H_{0}=73 \pm 3$ (random) $\mathrm{km} \mathrm{s}^{-1} \mathrm{Mpc}^{-1}$ for a sample of 17 Seyfert 1 galaxies observed with the MAGNUM telescope. We suggest that this method can be used with QSOs to study the dark energy in the universe beyond what is possible with type Ia supernovae.

We thank the staff at the Haleakala Observatories for their help with facility maintenance. This research has been supported partly by the Grants-in-Aid of Scientific Research $(10041110,10304014,11740120,12640233,14047206$, 14253001, 14540223, 25287062, and 16740106) and the COE Research (07CE2002) of the Ministry of Education, Science, Culture and Sports of Japan.

\section{REFERENCES}

Antonucci, R. 1993, ARA\&A, 31, 473

Barvainis, R. 1987, ApJ, 320, 537

Barvainis, R. 1990, ApJ, 353, 419

Bentz, M. C., Denney, K. D., Grier, C. J., et al. 2013, ApJ, 767, 149

Birkinshaw, M. 1999, PhR, 310, 97

Blandford, R., \& Narayan, R. 1986, ApJ, 310, 568

Cackett, E. M., Horne, K., \& Winkler, H. 2007, MNRAS, 380, 669

Collier, S., Horne, K., Wanders, I., \& Peterson, B. M. 1999, MNRAS, 302, L24

Czerny, B., Hryniewicz, K., Maity, I., et al. 2013, A\&A, 556, A97

Davis, S. W., Woo, J.-H., \& Blaes, O. M. 2007, ApJ, 668, 682

Draine, B. T., \& Lee, H. M. 1984, ApJ, 285, 89

Freedman, W. L., \& Madore, B. F. 2010, ARA\&A, 48, 673

Freedman, W. L., Madore, B. F., Gibson, B. K., et al. 2001, ApJ, 553, 47

Gaskell, C. M., Goosmann, R. W., Antonucci, R. R. J., \& Whysong, D. H. 2004, ApJ, 616, 147

Hubble, E. 1929, PNAS, 15, 168

Huffman, D. R. 1997, AdPhy, 26, 129

Kawaguchi, T., \& Mori, M. 2011, ApJ, 737, 105

Kishimoto, M., Antonucci, R., Blaes, O., et al. 2008, Natur, 454, 492

Kishimoto, M., Hönig, S. F., Beckert, T., \& Weigelt, G. 2007, A\&A, 476, 713

Kobayashi, Y., Sato, S., Yamashita, T., Shiba, H., \& Takami, H. 1993, ApJ, 404, 94

Kobayashi, Y., Yoshii, Y., Peterson, B. A., et al. 1998, Proc. SPIE, 3354, 769

Koshida, S., Yoshii, Y., Kobayashi, Y., et al. 2009, ApJL, 700, L109

Krawczyk, C. M., Richards, G. T., Mehta, S. S., et al. 2013, ApJS, 206, 4

Landt, H., Elvis, M., Ward, M. J., et al. 2011, MNRAS, 414, 218

Laor, A., \& Draine, B. T. 1993, ApJ, 402, 441 
Mathis, J. S., Rumpl, W., \& Nordsieck, K. H. 1997, ApJ, 217, 425

Minezaki, T., Yoshii, Y., Kobayashi, Y., et al. 2004, ApJL, 600, L35

Minezaki, T., Yoshii, Y., Kobayashi, Y., et al. 2006, ApJL, 643, L5

Mould, J. R., Huchra, J. P., Freedman, W. L., et al. 2000, ApJ, 529, 786 (with an erratum)

Oknyanskij, V. L., \& Horne, K. 2001, in ASP Conf. Ser. 224, Probing the Physics of Active Galactic Nuclei, ed. B. M. Peterson, R. W. Pogge, \& R. S. Polidan (San Francisco, CA: ASP), 149

Planck Collaboration et al. 2014, A\&A, in press (arXiv:1303.5076)

Sakata, Y., Minezaki, T., Yoshii, Y., et al. 2010, ApJ, 711, 461

Salpeter, E. E. 1977, ARA\&A, 15, 267

Schlegel, D. J., Finkbeiner, D. P., \& Davis, M. 1998, ApJ, 500, 525

Suganuma, M., Yoshii, Y., Kobayashi, Y., et al. 2006, ApJ, 639, 46

Telfer, R. C., Zheng, W., Kriss, G. A., \& Davidsen, A. F. 2002, ApJ, 565, 773
Tomita, H. 2005, Doctoral Dissertation, The University of Tokyo Tomita, H., Yoshii, Y., Kobayashi, Y., et al. 2006, ApJL, 652, L13

Tonry, J. L. 2000, ApJ, 530, 625

Urry, C. M., \& Padovani, P. 1995, PASP, 107, 803

Uttley, P. 2006, in ASP Conf. Ser. 360, AGN Variability from X-Rays to Radio Waves, ed. C. M. Gaskell, I. M. McHardy, B. M. Peterson, \& S. G. Sergeev (San Francisco, CA: ASP), 101

Vanden Berk, D. E., Richards, G. T., Bauer, A., et al. 2001, AJ, 122, 549

Watson, D., Denney, K. D., Vestergaard, M., \& Davis, T. M. 2011, ApJL, 740, L49

Winkler, H. 1997, MNRAS, 292, 273

Yoshii, Y. 2002, in New Trends in Theoretical and Observational Cosmology, ed. K. Sato \& T. Shiromizu (Tokyo: Universal Academy Press), 235

Yoshii, Y., Kobayashi, Y., \& Minezaki, T. 2003, BAAS, 35, 752 